\title{
miRNA let-7e targeting MMP9 is involved in adipose- derived stem cell differentiation toward epithelia
}

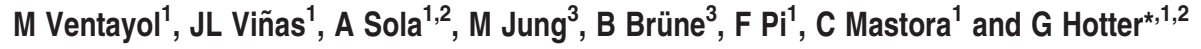

miRNA let-7e is involved in stem cell differentiation, and metalloproteinases are among its potential target genes. We hypothesized that the inhibitory action of let-7e on regulation of MMP9 expression could represent a crucial mechanism during differentiation of adipose-derived stem cells (ASCs). ASCs were differentiated with all-trans retinoic acid (ATRA) to promote differentiation, and the effect of let-7 silencing during differentiation was tested. Results indicate that ASCs cultured with ATRA differentiated into cells of the epithelial lineage. We found that ASCs cultured with ATRA or transfected with miRNA let-7e expressed epithelial markers such as cytokeratin-18 and early renal organogenesis markers such as Pax2, Wt1, Wnt4 and megalin. Conversely, the specific knockdown of miRNA let-7e in ASCs significantly decreased the expression of these genes, indicating its vital role during the differentiation process. Using luciferase reporter assays, we also showed that MMP9 is a direct target of miRNA let-7e. Thus, our results suggest that miRNA let-7e acts as a matrix metalloproteinase-9 (MMP9) inhibitor and differentiation inducer in ASCs.

Cell Death and Disease (2014) 5, e1048; doi:10.1038/cddis.2014.2; published online 6 February 2014

Subject Category: Experimental Medicine

Adipose-derived stem cells (ASCs) are attractive targets for clinical applications. They offer multiple advantages such as good accessibility, abundance and the ability to expand in culture, maintaining their multilineage differentiation potential. ${ }^{1,2}$ Under the appropriate stimulus, ASCs differentiate into cells with characteristics of hepatocytes, ${ }^{3}$ neurons, ${ }^{4}$ endothelial cells ${ }^{5}$ and/or renal epithelial cells. ${ }^{6}$

ASCs mainly express a mesenchymal cellular phenotype, and their differentiation toward cells with epithelial characteristics requires extensive alterations in gene expression. Interestingly, it has been shown that the in vitro process of differentiation toward renal epithelial cells is phenotypically almost identical to the one that occurs during renal organogenesis, in terms of both morphology and specific protein expression patterns. ${ }^{7}$ Differentiation of organs demands a mesenchymal-to-epithelial transition (MET), a core mechanism that allows cells to acquire a specific lineage-determined phenotype. Cellular changes associated with MET include major changes in the expression of several genes related to the conversion of the extracellular matrix, ${ }^{8}$ such as matrix metalloproteinases ${ }^{9,10}$ and the redistribution of the cytoskeletal structure, finally leading to the establishment of the typical apical-basal polarity of epithelial cells. These morphological changes result from a specific expression profile of surface markers, transcription factors and microRNAs (miRNAs).

The miRNAs are small ( $\sim 22 \mathrm{nt})$, regulatory, noncoding RNA molecules that control the expression of their target
mRNAs at the post-transcriptional level by binding to the $3^{\prime}$ untranslated region ( $\left.3^{\prime} \cup T R\right)$. There is a well-established biotechnology system for studying the role and targets of miRNAs in vitro and also in vivo. Using synthetic miRNAs and transfection methods, we can mimic or suppress the action of specific miRNAs and study the consequences in different models. The miRNAs are involved in the differentiation of diverse cell lineages-among others, skeletal muscle cells, ${ }^{11}$ cardiomyocytes ${ }^{12}$ and neurons. ${ }^{13}$ Interestingly, critical signaling pathways during differentiation, such as the Wnt pathway, ${ }^{14}$ are mainly controlled by the expression of specific miRNAs. miRNA family let-7 (let-7) is considered as a global regulator of differentiation. ${ }^{15}$ The let-7 is barely detected during embryonic development, but it increases significantly as tissues differentiate and it is mainly detected in the adult kidney. ${ }^{16}$ In addition, let-7e plays a pivotal role in stem cell differentiation, ${ }^{17,18}$ and its loss results in reversion of embryogenesis and dedifferentiation. Decreased levels of miRNA let-7e have also been associated with metastasis and poor prognosis in renal cell carcinoma, ${ }^{19}$ possibly because of its effects on matrix metalloproteinases (MMPs). It has previously been shown that miRNA let-7b downregulates MMP9 activity through indirect effects by inhibition of basigin. ${ }^{20,21}$ By using in silico analysis of the $3^{\prime}$ UTR of MMP9, a predicted binding site of miRNA let-7e was indicated. ${ }^{22}$ Nevertheless, the direct effect of miRNA let-7e on MMP9 expression has not been investigated to date.

\footnotetext{
${ }^{1}$ Department of Experimental Pathology, Instituto de Investigaciones Biomédicas de Barcelona, Spanish Research Council (IIBB-CSIC, IDIBAPS), Barcelona, Spain; ${ }^{2}$ CIBER-BBN, Networking Centre on Bioengineering, Biomaterials and Nanomedicine (CIBER-BBN), Department of Experimental Pathology, IIBB-CSIC-IDIBAPS, Barcelona, Spain and ${ }^{3}$ Institute of Biochemistry I/ZAFES, Goethe-University Frankfurt, Theodor-Stern-Kai 7, Frankfurt, Germany

${ }^{*}$ Corresponding author: G Hotter, Department of Experimental Pathology, (IIBB-CSIC-IDIBAPS), C/Rosselló, 161, 7th Floor, 08036 Barcelona, Spain. Tel: +34 93 3638334; Fax: +34 93 3638300; E-mail: ghcbam@iibb.csic.es

Keywords: adipose-derived stem cells (ASCs); miRNA let-7e; MMP9; differentiation

Abbreviations: ASCs, adipose-derived stem cells; ATRA, all-trans retinoic acid; MMP9, matrix metalloproteinase-9; MET, mesenchymal-to-epithelial transition; miRNA, microRNA; UTR, untranslated region; CK18, cytokeratin-18; E-CAD, E-cadherin

Received 12.7.13; revised 09.10.13; accepted 25.11.13; Edited by Y Shi
} 
Thus, as miRNA let-7e adopts a pivotal role during stem cell differentiation, and MMP9 may be a potential direct let-7e target, we hypothesized that the inhibitory action of let-7e on the regulation of MMP9 expression could represent a crucial mechanism during renal organogenesis. In this study we show that let-7e is induced during renal epithelial cell differentiation from ASCs and that the direct inhibition of MMP9 by miRNA let-7e plays a key role during this process.

\section{Results}

ASCs have multilineage potential and phenotypical characteristics of mesenchymal stem cells. ASCs obtained from male Swiss CD1 mice were isolated and expanded in vitro in control media. In order to confirm their multilineage potential, these cells were cultured with adipogenic media for 15 days and with osteogenic media for 20 days. ASCs cultured under adipogenic conditions formed lipid vacuoles that are considered to be typical features of the adipogenic lineage (Figure 1a, left panel). ASCs cultured under osteogenic conditions showed depositions of mineralized matrix, a characteristic of the osteogenic lineage
(Figure 1a, right panel). These observations confirmed the multilineage potential of ASCs. Cellular composition was analyzed by flow cytometry (Figure 1b). Approximately $90 \%$ of the population was positive for the mesenchymal stem cell markers CD44, Sca-1 and CD29, and negative for CD34 and CD45.

ATRA promotes morphological differentiation of ASCs. Phase-contrast microscopy analysis of ASCs cultured with $5 \mu \mathrm{M}$ all-trans retinoic acid (ATRA) for 11 days showed a gradual conversion into a more epithelial polygonal phenotype with respect to untreated controls (Figure 1c, upper panel). A flattened fibroblast-like morphology was observed in untreated cells, whereas ATRA stimulation gradually changed cell morphology into an epithelial, cobblestone-like phenotype. Changes in cytoskeletal organization were assessed by immunofluorescence staining of the actin fiber network (Figure 1c, lower panel). Cells showed an organized cytoskeletal network with dense actin fibers at the edges when cultured with ATRA for 11 days. Untreated controls maintained the equal actin fiber distribution throughout the whole cell, typical of the mesenchymal phenotype.

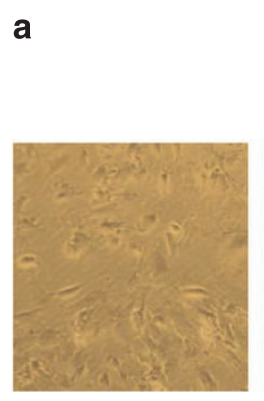

Control

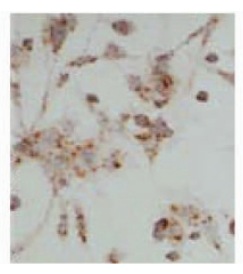

Adipogenic

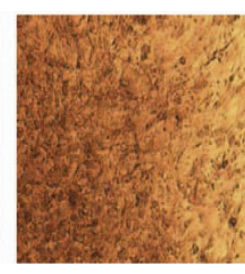

Osteogenic b
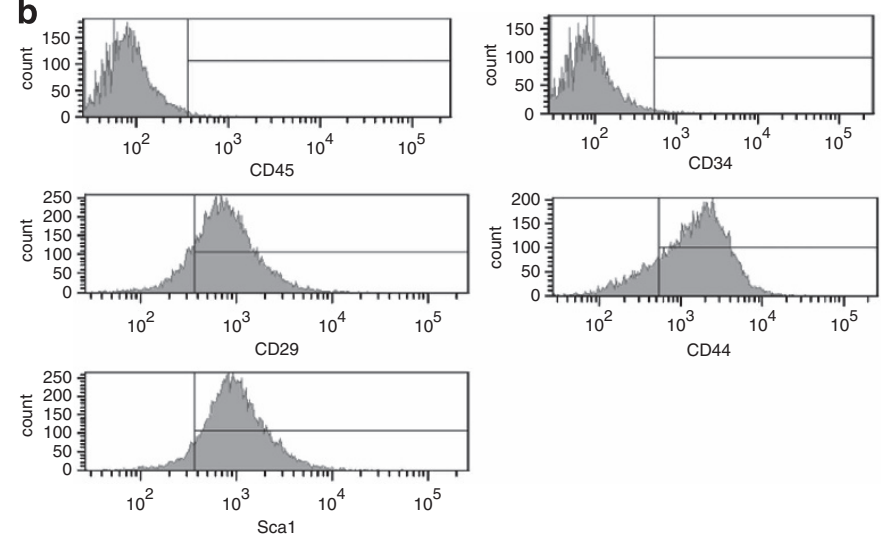

Osteogenic
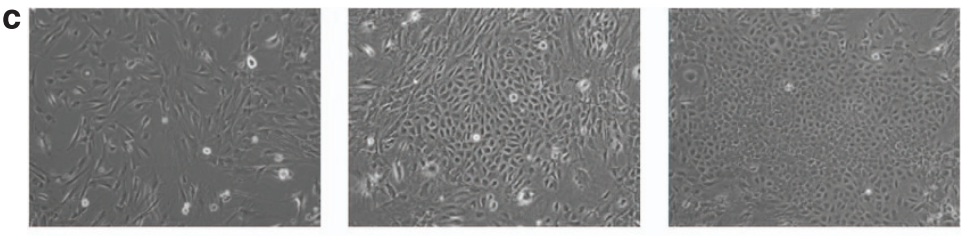

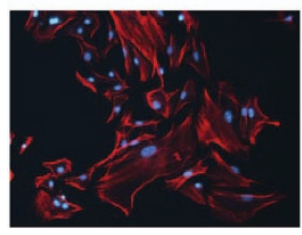

Untreated

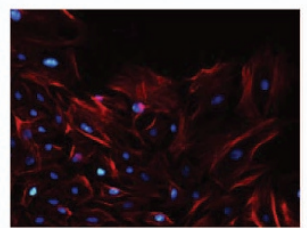

DAY 6

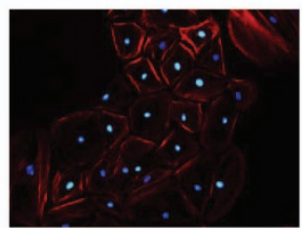

DAY 11

Figure 1 Multilineage potential and mesenchymal stem cell phenotype of mouse ASCs. (a) Oil red staining after 15 days of culture in adipogenic media (left panel). A representative image from five independent experiments is illustrated. Magnification $\times 20$. Alizarin red staining after 20 days of culture in osteogenic media (right panel). A representative image from five independent experiments is illustrated. Magnification $\times 20$. (b) ASCs were positive for Sca-1, CD44 and CD29, and negative for CD34 and CD45, confirming their mesenchymal origin. (c) Morphological representation of mesenchymal-to-epithelial transition. Phase-contrast images representative of ASC morphology change during the differentiation protocol. ASCs were left untreated or differentiated with ATRA for 6 or 11 days (upper panel). Representative images from four independent experiments are illustrated. Magnification $\times 20$. Phalloidin staining of f-actin cytoskeleton (in red) contributes to visualize the epithelial-like morphology (day 11 ) in contrast to the more fibroblast-like morphology of untreated, undifferentiated cells. Nuclei were stained with DAPI (in blue) and images obtained by fluorescence microscopy. A representative picture from four independent experiments is shown. Magnification $\times 40$ 
ATRA promotes differentiation of ASCs toward the epithelial lineage. Upregulation of $\mathrm{Pax} 2$, an intermediate mesoderm marker, and also upregulation of WT1, Wnt4, Six2 and megalin, all markers of the metanephric mesenchyme, were observed when ASCs were cultured in media with a physiological concentration of glucose and supplemented with ATRA (Figure 2).

ASCs also showed a significant induction of the epithelial markers cytokeratin-18 (CK18) and E-cadherin (E-CAD), whereas mRNA expression of vimentin, a classical mesenchymal marker, was downregulated (Figure 3). Expression of CK18 was also confirmed by immunocytochemistry (Figure 3). CK18 appeared as a perinuclear brown staining when ASCs were cultured for 11 days under differentiation conditions. In contrast, untreated ASCs showed no CK18 staining.

miRNA let-7e is upregulated in ASC differentiation and is involved in the expression of early nephrogenic and epithelial markers. In order to evaluate the role of miRNA let-7e in ASC differentiation, ASCs were transfected with $100 \mathrm{nM}$ miRNA let-7e at day 2 of culture and collected at day 6 .

ASCs were also differentiated with ATRA and transfected with anti-miRNA let-7e for knockdown at day 4 and collected at day 6 . As we wanted to test the effect of let7 e knockdown on differentiation markers, which did not show a significant increase until day 4 of differentiation (see Figure 2), this particular time point was chosen to deliver anti-miRNA let-7e. A schematic overview is given in Figure 4.

The expression of miRNA let-7e (Figure 4) and nephrogenic markers (Figure 5) was evaluated. The miRNA let-7e transfection significantly upregulated let-7e expression and induced the expression of the metanephric mesenchyme markers Pax2, Wnt4, Wt1 and megalin as well as the epithelial marker CK18. In contrast, miRNA let-7e knockdown blocked the increase in early nephrogenic and epithelial markers observed during ASC differentiation with ATRA.
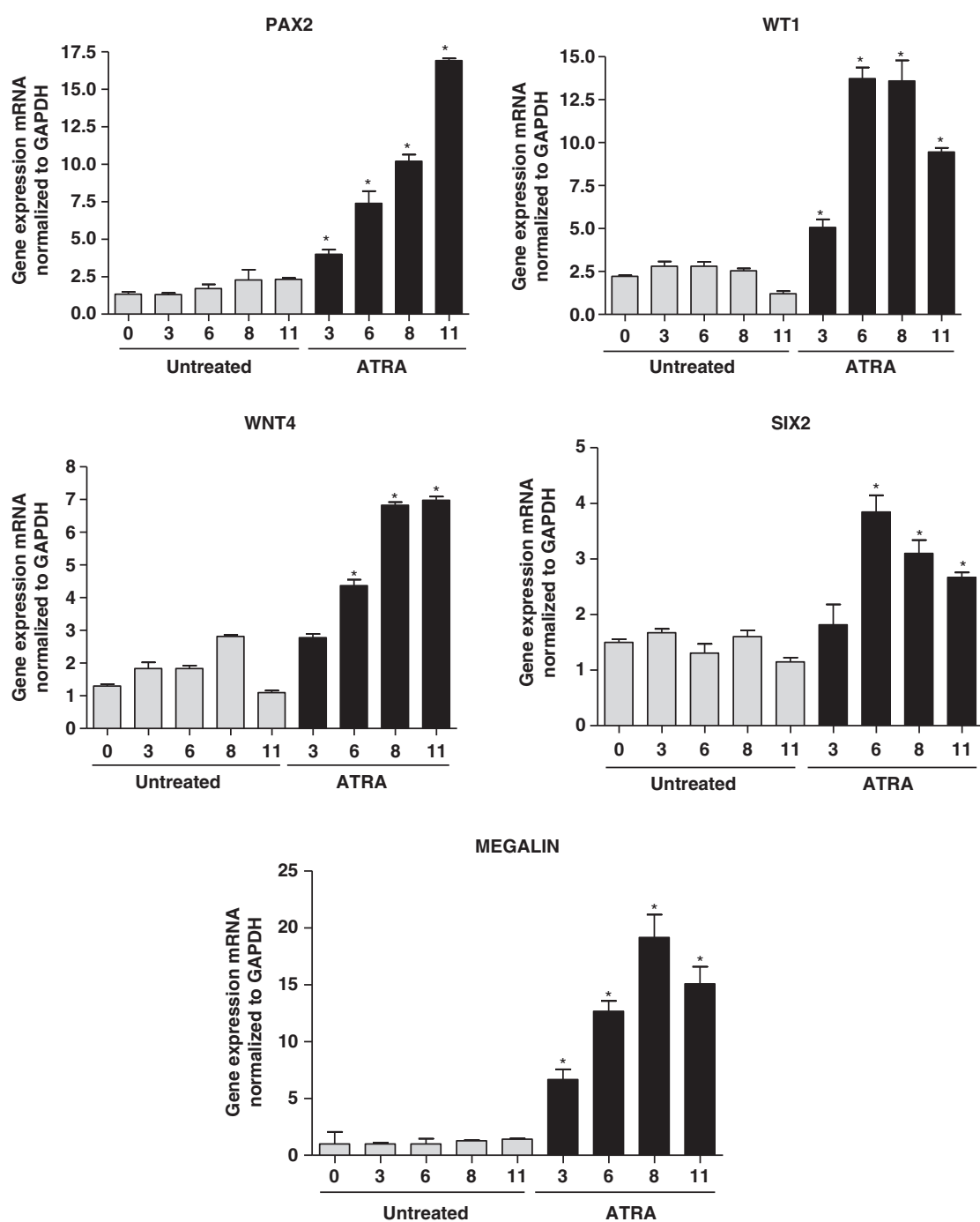

Figure 2 ATRA stimulation of ASCs promotes overexpression of early nephrogenic markers. qRT-PCR analysis of early nephrogenic markers PAX2, WT1, Wnt4, Six2 and megalin. Total RNA was extracted from ASCs cultured for 1 to 11 days with ATRA (black columns) or with control medium (gray columns). A sample of ASCs on day 0 was analyzed as control; $n=4$. Data are means \pm S.D. ${ }^{*} P<0.05$ versus untreated 
CK18

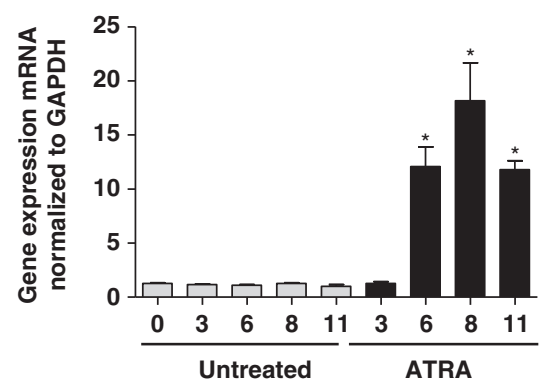

E-CAD

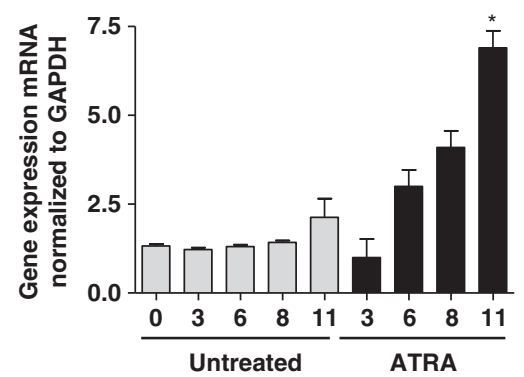

VIMENTIN
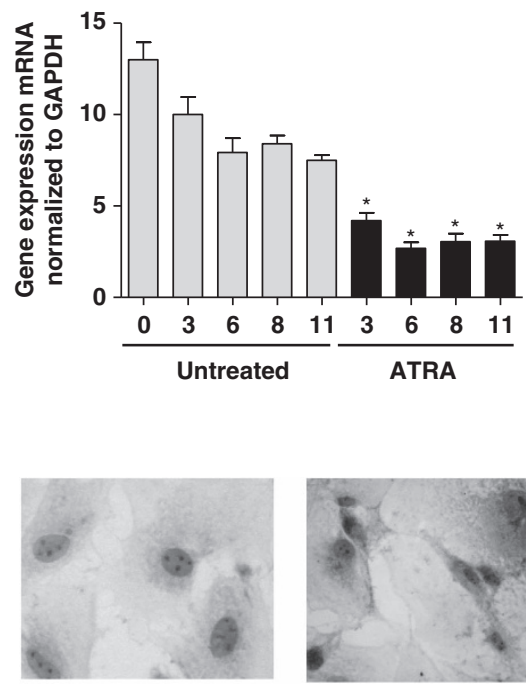

Untreated

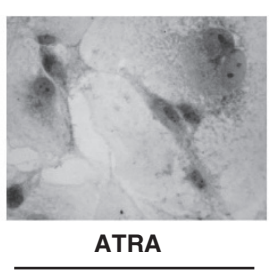

Figure 3 ATRA stimulation of ASCs promotes epithelial differentiation. qRT-PCR analysis of epithelial markers CK18 and E-CAD and the mesenchymal marker vimentin. Total RNA was extracted from ASCs cultured for 1 to 11 days with ATRA (black columns) or with control medium (gray columns). A sample of ASCs on day 0 was analyzed as control. $n=4$. Data are means \pm S.D. ${ }^{*} P<0.05$ versus untreated. Immunocytochemistry of the epithelial marker CK18. A perinuclear gray area in ASCs cultured for 11 days was observed after incubation with ATRA (day 11). No staining was observed in untreated cells. Nuclei were hematoxylin stained. A representative image from four independent experiments is shown. Magnification $\times 40$

miRNA let-7e targets the $3^{\prime}$ UTR of MMP9. The overexpression of miRNA let-7e in ASCs cultured under untreated conditions provoked the downregulation of MMP9 mRNA expression (Figure 6a). MMP9 activity was also reduced, as observed in zymographic gels. Both pro-form and active MMP9 were downregulated compared with untreated controls (Figure 6b).

In order to determine whether MMP9 was regulated by let$7 e$ through direct binding of the miRNA to the $3^{\prime} U T R$, we transfected either a control vector (control), a wild-type construct ( $3^{\prime}$ UTR-MMP9) with an intact let-7e-binding site or the mutated construct ( $3^{\prime}$ UTR-mutMMP9) in HEK293 cells (Figure 6c). We chose HEK293 because this cell type can be transfected very easily and is a good starting point for experiments that include luciferase constructions and co-transfection with miRNAs. Cells were co-transfected with either control miRNA (C) or miRNA let-7e (+let-7e). Luciferase activity showed a significant decrease in cells treated with let-7e, suggesting that let-7e binds to the $3^{\prime}$ UTR of MMP9. In contrast, the mutation of the let-7e-binding site in the $3^{\prime}$ UTR of MMP9 led to an increase in luciferase activity, confirming the involvement of let-7e in MMP9 inhibition.

Similar results were found using ASCs under untreated conditions (Figure 6d). Co-transfection of let-7e in undifferentiated ASCs reduced luciferase activity using the wild-type reporter construct for the $3^{\prime} U T R$ of MMP 9 containing the intact let-7e-binding site. To confirm these data, we used ASCs differentiated with ATRA for 8 days and co-transfected the cells with either anti-let-7e or a control antimiR. The data showed a significant increase in luciferase activity by blocking let-7e during differentiation. These results further corroborate the notion that let-7e has a regulatory effect on MMP9 expression.

We also checked whether the increased expression of MMP9 provoked by miRNA let-7e silencing was directly correlated with MMP9 activity. In situ zymography experiments were performed and gelatinolytic activity was measured according to the levels of fluorescence emitted through digestion of FITC-labeled gelatine (Figure 7a). The results showed increased gelatinase activity when miRNA let-7e was blocked. Finally, we tested the role of MMP9 on epithelial cell features by assessing E-cadherin expression (Figure 7b). Both ASCs (left panel) and NRK-52e cells, an established kidney tubular epithelial cell line (right panel), were used to detect E-cadherin expression by western blot analysis. ASCs treated with ATRA showed a significant increase in E-cadherin expression compared with untreated cells. This increase was blocked by the addition of recombinant MMP9. The same results were obtained when using NRK-52e cells, thus confirming the inhibitory effect of MMP9 on epithelial cell characteristics.

\section{Discussion}

ASCs are pluripotent cells that can differentiate mainly into cells of the same lineage commitment, such as osteocytes, chondrocytes and adipocytes. ${ }^{23}$ Nevertheless, various studies proposed that ASCs can overcome this lineage commitment and differentiate into renal epithelia. Renal epithelial differentiation of ASCs was first observed after transplantation of human ASCs into ischemic mouse kidneys. ASCs were readily integrated into the tissue and differentiated 


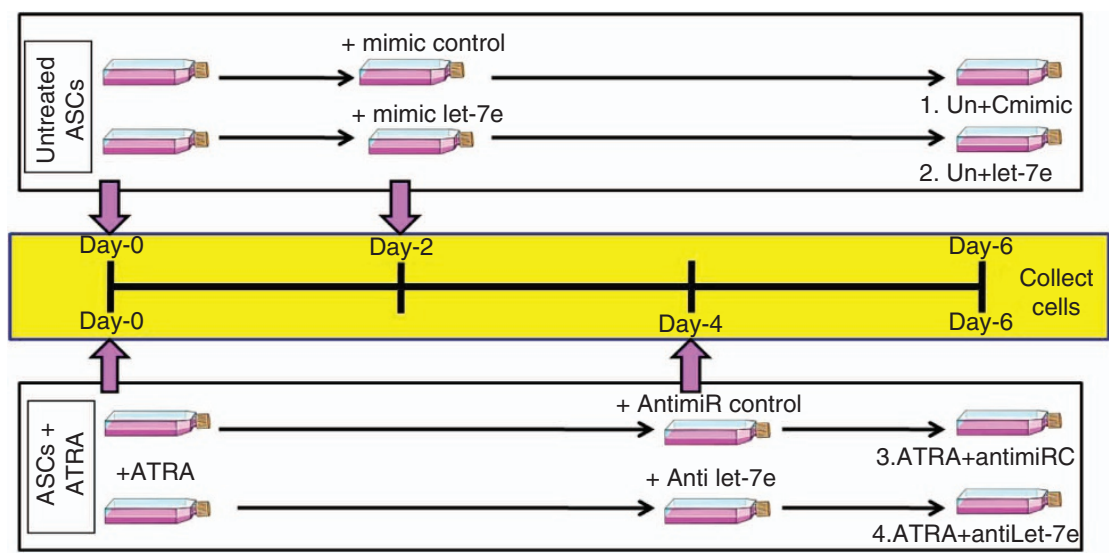

miRNA Let-7e

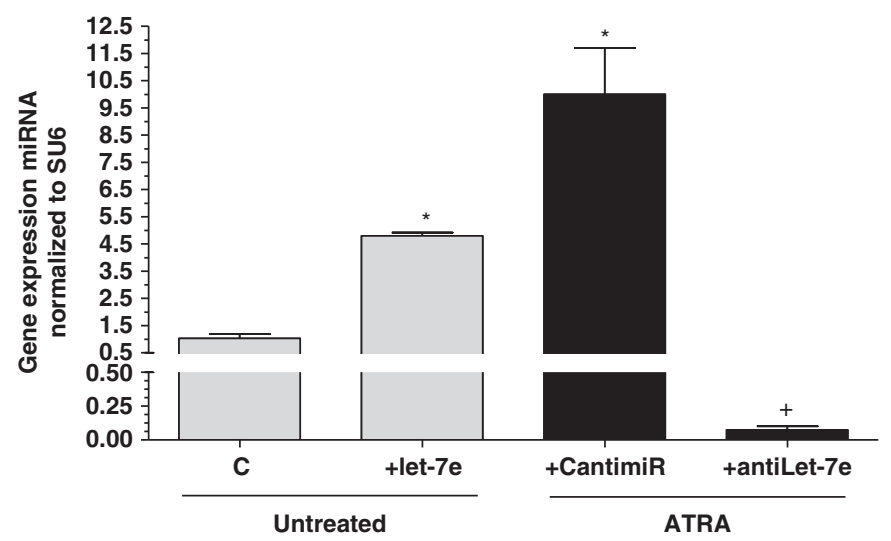

Figure 4 miRNA let-7e is upregulated during differentiation. (Upper) Schematic overview of treatments. Silencing and mimicking miRNA let-7e protocol used in ASC culture. Untreated ASCs were transfected with $100 \mathrm{nM}$ let-7e or control at day 2 of differentiation, and collected at day 6 . ATRA-treated cells were transfected with $100 \mathrm{nM}$ antiLet-7e or control anti-miR (CantimiR) at day 4 and collected at day 6. (Lower) Total RNA was extracted from ASCs with or without ATRA and transfected with let-7e, a silencing oligonucleotide (antiLet-7) or their respective negative controls (C/CantimiR). Samples were assayed for miRNA let-7e expression by qRT-PCR and normalized to SU6; $n=4$. Data are means \pm S.D. ${ }^{*} P<0.05$ versus $C$ mimc; ${ }^{+} P<0.05$ versus CantimiR

into epithelial-like cells, as corroborated by the expression of CK18. ${ }^{6}$ In vitro studies also showed ASC differentiation toward renal epithelium that could be induced by factors secreted from renal tubular epithelial cells. ${ }^{24,25}$

Moreover, Brzoska et al. ${ }^{26}$ described an in vitro protocol using ATRA in order to differentiate ASCs into epithelial cells that expressed increased levels of the epithelial marker CK18 as well as decreased amounts of the mesenchymal marker vimentin.

In the present study, ASCs cultured with ATRA gradually increased the expression of different early nephrogenic markers, such as Pax2, Wt1, Wnt4, Six2 and megalin. At the same time, epithelial markers CK18 and E-CAD were upregulated, whereas the mesenchymal marker vimentin was significantly decreased (Figures 2 and 3). Most interestingly, stem cell differentiation reflected the process of kidney organogenesis during development. Therefore, in vitro differentiation of stem cells might be expected to mimic the expression pattern of differentiation markers of the development from the intermediate mesoderm to the metanephric mesenchyme, and finally to the renal epithelium. ATRA treatment increases the expression of Pax2, one of the first genes expressed during kidney embryogenesis in the intermediate mesoderm and the first indicator of renal lineage determination. ${ }^{27}$ ATRA-treated cells also express Wt1 and Six $2,{ }^{28}$ which are the main transcription factors expressed in nephron precursors, and are essential for correct kidney formation during development. Wt1 is readily upregulated in the metanephric mesenchyme together with Wnt4, ${ }^{29}$ a component of the Wnt pathway, and both play a crucial role during the transition of the mesenchyme to renal epithelia. ${ }^{30}$ Furthermore, the membrane glycoprotein megalin adopts a pivotal role in determining kidney functional responses in renal epithelial cells. Stem cell differentiation may also be monitored by determining epithelial versus mesenchymal markers. During in vitro differentiation and MET, epithelial markers CK18 Griesche et al. ${ }^{31}$ and E-CAD ${ }^{32}$ were described to be upregulated, whereas mesenchymal markers such as vimentin ${ }^{33}$ were concomitantly downregulated. After treatment with ATRA, cells undergo significant morphological changes. The typical fibroblastic, mesenchymal-like morphology of ASCs gradually changes toward a more epithelial-like, polygonal morphology. These morphological and cytoskeletal changes represent the key process during MET, allowing the cells to adopt the characteristic epithelial cell apical-basal polarity. 


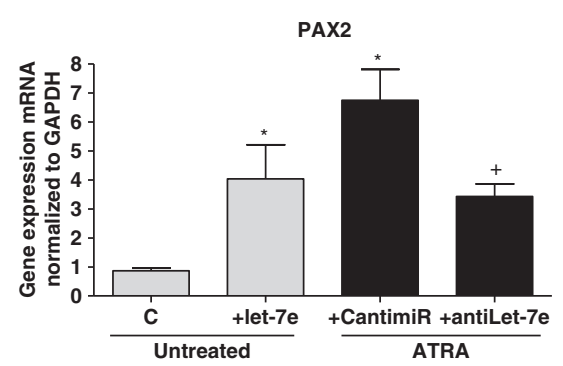

MEGALIN
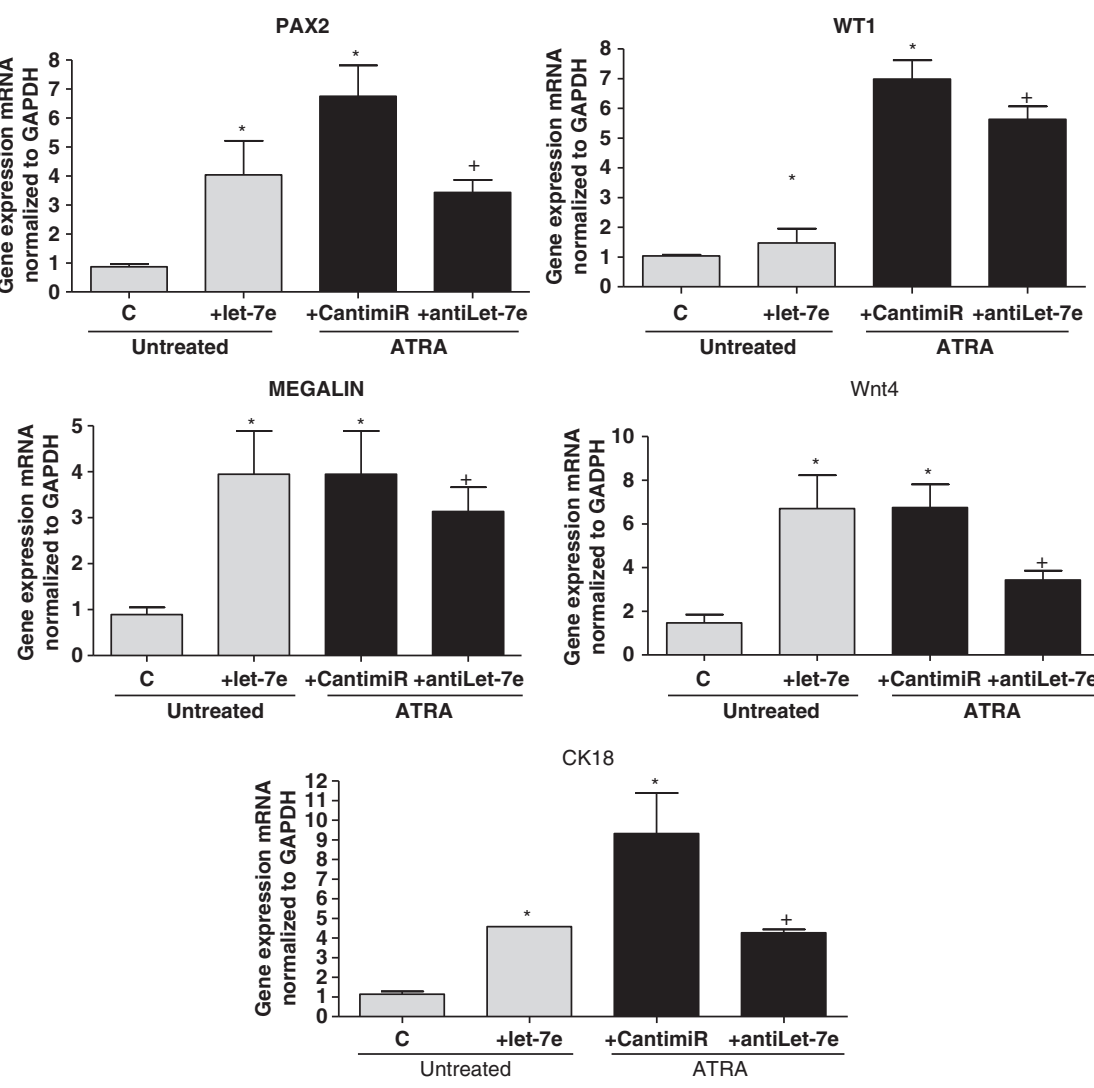

Wnt4
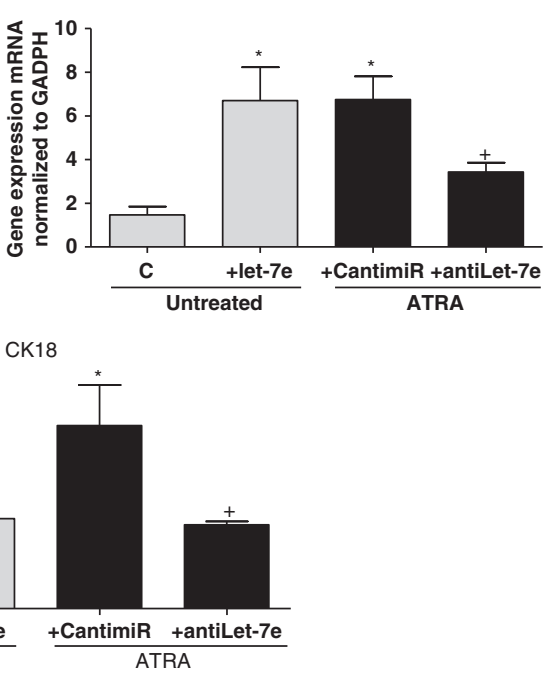

Figure 5 miRNA let-7e influences the expression of early nephrogenic and epithelial markers. ATRA-treated cells were transfected with $100 \mathrm{nM}$ antiLet-7e or control antimir (CantimiR) at day 4 and collected at day 6. RT-PCR of ASCs transfected with $100 \mathrm{nM}$ let-7e shows increased expression of PAX2, Wnt4 and WT1 compared with control cells (C). miRNA let-7e silencing ( + antiLet-7e) reduces the expression of early nephrogenic markers PAX2, Wnt4, WT1, megalin and CK18 compared with control antimiR-transfected cells $\left(+\right.$ CantimiR); $n=4$. Data are means \pm S.D. ${ }^{*} P<0.05$ versus Cmimc; ${ }^{+} P<0.05$ versus CantimiR. mRNA expression levels were normalized to GAPDH

The let-7 family of miRNAs is classically involved in stem cell differentiation. ${ }^{17,18}$ It was previously shown that miRNA let-7, together with miRNA-24, adopts a specific role in osteogenic differentiation through the PDGF pathway. ${ }^{17}$ Interestingly, miRNA let-7 is barely detectable in the embryonic kidney, but is significantly expressed in the adult kidney. ${ }^{16,34}$ Moreover, let-7 expression is clearly decreased in pathophysiological states of cellular dedifferentiation such as renal cell carcinoma. ${ }^{19}$ In our study, we were able to show clearly that miRNA let-7e was significantly increased in ASCs differentiated with ATRA in vitro (Figure 4). This increase was concomitant with an increased expression of Pax2, Wt1, Wnt4, megalin and CK18. In contrast, blocking of miRNA let-7e in differentiated cells reversed these gene expression patterns, indicating the involvement of miRNA let-7e in renal epithelial cell differentiation.

MMPs play a key role in the differentiation of diverse tissues and organs throughout the body. ${ }^{35}$ During renal organogenesis, MMP2 and MMP9 are mainly synthesized by renal mesenchyma when the ureteric bud interacts with the metanephric mesenchyme. Interestingly, only MMP9, but not MMP2, was shown to play a crucial role during in vitro renal organogenesis. ${ }^{9}$ MMP9 degrades denatured collagen (gelatin), native collagen types I, IV, V and IX and elastin with high specificity. Therefore, it was speculated that MMP9 is involved in epithelial differentiation because of its role in extracellular matrix remodeling, a central step in MET. ${ }^{9}$ MMP9 also has the ability to degrade other nonstructural molecules such as E-CAD as well as other cell-to-cell adhesion molecules. ${ }^{36-38}$ Degradation of E-cadherin through MMP9 miRNAs promotes the process of epithelial-to-mesenchymal transition and cellular dedifferentiation. ${ }^{36}$ Our results further corroborate these findings, as addition of recombinant MMP9 to differentiated epithelial cells (from ASC or NRK-52e cell line) significantly reduced the protein levels of E-CAD. In this regard, the blocking of MMP9 may possibly be a key event in the maintenance of the epithelial phenotype. We conclude that MMPs are crucial players during differentiation in both MET and EMT. ${ }^{9}$ Therefore, there is a strong need for tight regulation of both the expression and activity of MMPs. Regarding the regulation of MMP9, miRNAs have been reported to act at the post-transcriptional level. ${ }^{39}$ Moreover, MMP9 mRNA is classified in the miRWalk database as a predicted target gene of miRNA let-7e ${ }^{22}$ and a recent report showed the indirect relationship of MMP9 and members of the let-7 family through regulation of the MMP inducer basigin. ${ }^{20}$ Our results point rather to a direct interaction of miRNA let-7e with MMP9 (Figure 6) shown by luciferase reporter assays using full-length constructs of the $3^{\prime}$ UTR of MMP9 and mutated constructs, deficient at the let-7e-binding site. 


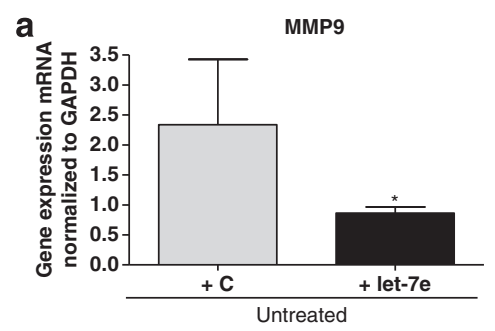

b
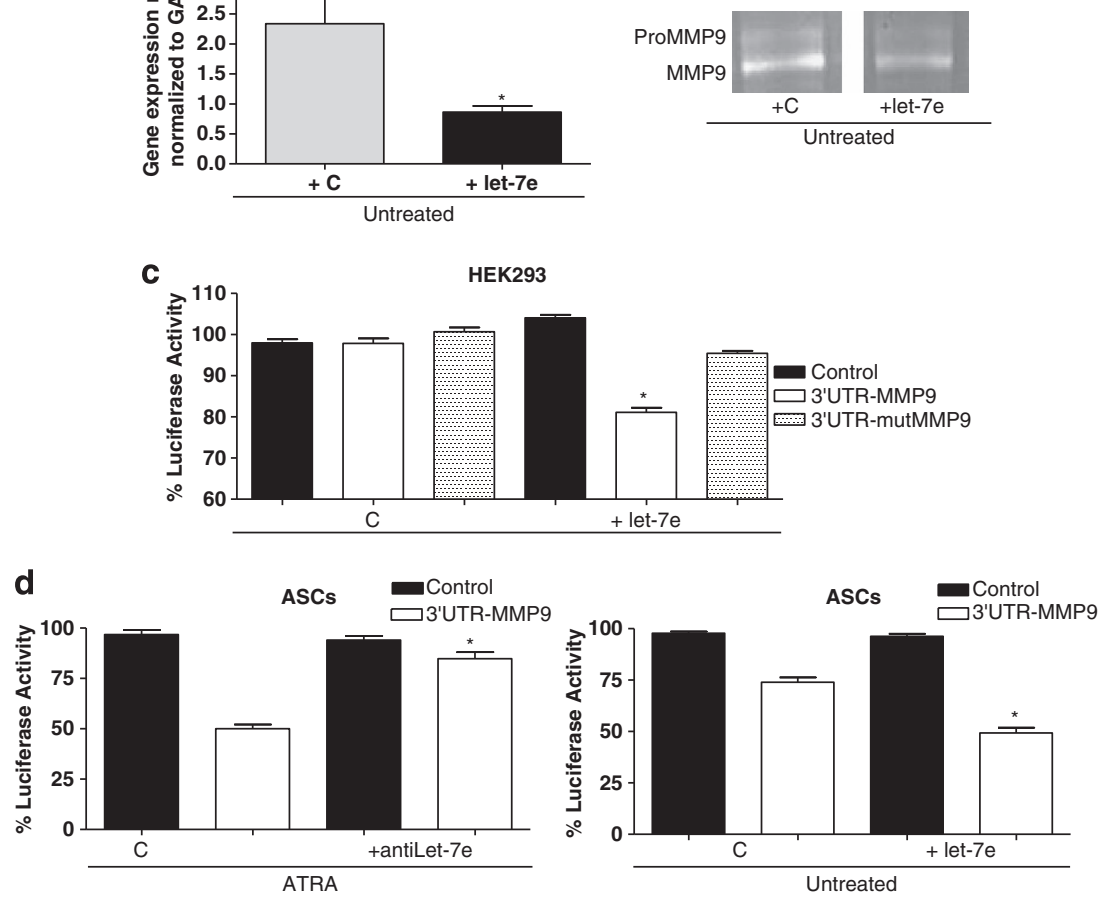

Figure 6 miRNA let-7e targets MMP9 in ASCs during differentiation. (a) Untreated ASCs transfected with $100 \mathrm{nM}$ let-7e ( + let-7e) downregulate the expression of MMP9 mRNA measured by qRT-PCR; $n=4$. Data were normalized to GAPDH. Results are expressed as mean \pm S.D. ${ }^{*} P<0.05$ versus + Control. (b) Gelatin zymography of supernatants $24 \mathrm{~h}$ after transfection with let-7e $(100 \mathrm{nM})$ or control oligonucleotides. (c) miRNA let-7e targets the mouse MMP9 3'UTR in HEK293 cells, (d) miRNA let-7e targets the mouse MMP9 $3^{\prime}$ UTR in untreated ASCs and in ASCs undergoing differentiation with ATRA. Cells were transfected either with the control vector (Control), a wildtype construct ( $\left.3^{\prime} U T R-M M P 9\right)$ with an intact let-7e-binding site or the mutated construct ( $3^{\prime} U T R$-mutMMP9), as indicated. Cells were co-transfected with either control miRNA (c), miRNA let-7e ( + let-7e), control antimiR (CantimiR) or anti-let-7e ( + antiLet-7), respectively. Relative luciferase activity was measured by determining firefly and Renilla luciferase. Data are expressed as $\%$ of luciferase activity; $n=4$. Data are means \pm S.D. ${ }^{*} P<0.05$ versus Control

In summary, we found that miRNA let-7e adopts a pivotal role during the differentiation of ASCs to epithelium. We further characterized MMP9 as a direct target of miRNA let$7 \mathrm{e}$ and obtained mechanistic insights into epithelial cell differentiation from ASCs. To conclude, miRNA let-7e induces ASC epithelial differentiation by modulating MMP9 expression.

\section{Materials and Methods}

Isolation and expansion of mouse ASCs. Male Swiss CD1 mice (Charles River, Wilmington, MA, USA) were used for the extraction of subcutaneous adipose tissue, as previously reported. ${ }^{40}$ All donors were $3-4$ months old and were killed by $\mathrm{CO}_{2}$ inhalation. ASCs were isolated from fat pads overlying the pelvis and proximal femurs based on standard protocols. ${ }^{41}$ Briefly, fat pads were excised, washed extensively with PBS containing $2 \%$ penicillin-streptomycin (Gibco, Life Technologies, Madrid, Spain) and $0.5 \mu \mathrm{g} / \mathrm{ml}$ amphotericin B (Gibco, Life Technologies) and then minced using a scalpel. Minced fat tissue was digested with collagenase Type IA (Sigma-Aldrich) for $1 \mathrm{~h}$ at $37^{\circ} \mathrm{C}$ and 150 r.p.m. Following digestion, the reaction was stopped with expansion medium and cell suspension was centrifuged ( $400 \times g, 10 \mathrm{~min})$. Floating material (adipocytes and fibrous material) was discarded, and the cell pellet (stromal vascular fraction) was resuspended and filtered through a $70 \mu \mathrm{m}$ cell strainer (BD Bioscience, Madrid, Spain). The stromal vascular fraction was filtrated and recovered by a second centrifugation $(400 \times \mathrm{g}, 7 \mathrm{~min})$. Pellet was resuspended in expansion medium and cultured in a T175 plastic flask (Greiner Bio-one, Frickenhausen, Germany). After $24 \mathrm{~h}$, nonadherent cells were removed and adherent cells containing ASCs were further expanded and passaged before reaching $80 \%$ confluence.
Expansion culture consisted of DMEM with physiologic glucose concentration (1000 mg/l; Sigma-Aldrich) supplemented with $10 \%$ fetal bovine serum (FBS) (Gibco, Life Technologies) and 1\% penicillin-streptomycin.

Multilineage differentiation potential: adipogenic and osteogenic differentiation protocol. For adipogenic differentiation, ASCs were cultured in 12-well plastic plates, seeded at a density of $22000 \mathrm{celll} / \mathrm{cm}^{2}$ and then cultured for 2 weeks in $\alpha$-MEM medium (PAA, Pasching, Austria) supplemented with $10 \% \mathrm{FBS}, 1 \mu \mathrm{M}$ dexamethasone (Sigma-Aldrich) and $320 \mathrm{nM}$ insulin (SigmaAldrich). ASCs were stained with $0.2 \%$ Oil Red $O$ reagent (Sigma-Aldrich) for lipid detection. ${ }^{41}$ For osteogenic differentiation, ASCs were cultured in 12-well plastic plates seeded at a density of $4200 \mathrm{cells} / \mathrm{cm}^{2}$ and then cultured for 20 days in $\alpha$-MEM medium (PAA) supplemented with $10 \%$ FBS, $10 \mathrm{nM}$ dexamethasone (Sigma-Aldrich), $200 \mu \mathrm{M}$ ascorbate-2-phosphate (Sigma-Aldrich) and $10 \mathrm{mM}$ $\beta$-glycerophosphate (Sigma-Aldrich). ASCs were stained with $2 \%$ Alizarin red (Sigma-Aldrich) to detect matrix mineralization. ${ }^{23}$

Flow cytometry. Flow cytometric characterization of ASCs (passage 3) was performed as previously reported ${ }^{42,43}$ for the surface markers Sca-1, CD 44, CD29, CD45 and CD34. These markers were selected in accordance with previously published studies. ${ }^{44,45}$ Briefly, ASCs were trypsinized, washed with PBS and centrifuged at $400 \times g$ for 5 min. Cell aliquots $\left(2 \times 10^{5}\right)$ were then blocked with goat serum for $30 \mathrm{~min}$ at $4^{\circ} \mathrm{C}$ and resuspended in $100 \mu \mathrm{l}$ of PBS/BSA buffer. Finally, anti-mouse antibodies FITC-Sca1 (AbD Serotec, Oxford, UK), FITC-CD29 (AbD Serotec), FITC-CD34 (AbD Serotec), PE-CD44 (Southern Biotech, Birmingham, AL, USA), Alexa fluor700-CD45 (AbD Serotec) and their respective isotype controls were added and incubated for $30 \mathrm{~min}$ at RT. Propidium iodide (PI) staining (Sigma-Aldrich) was performed $(1 \mu \mathrm{g} / \mathrm{ml})$ in order to check cell viability. Detection was performed on a FACS canto II cytometer (BD Bioscience). 

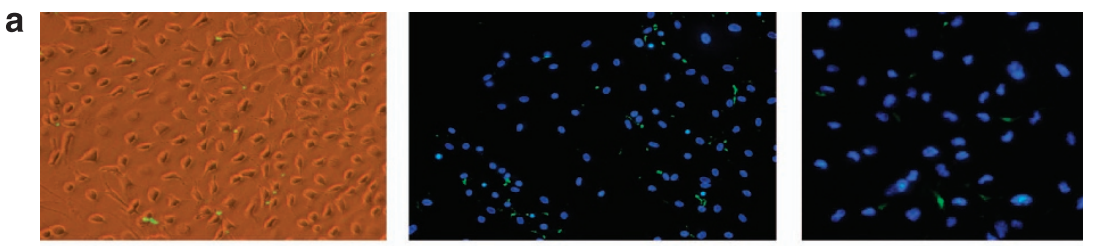

ATRA+CantimiR

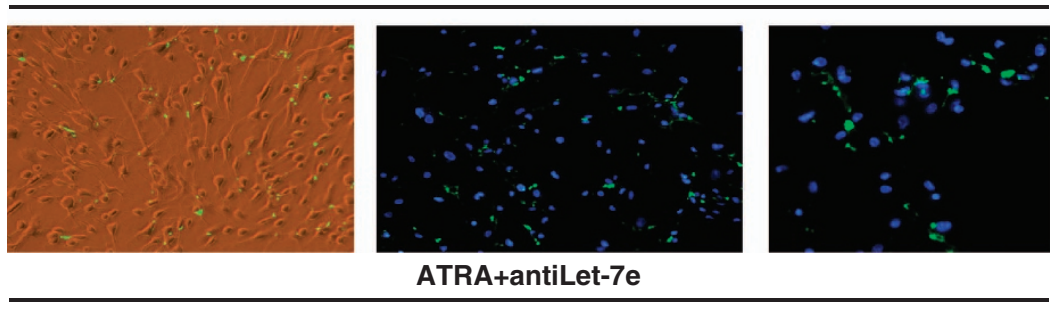

Gelatinase activity

nuclei

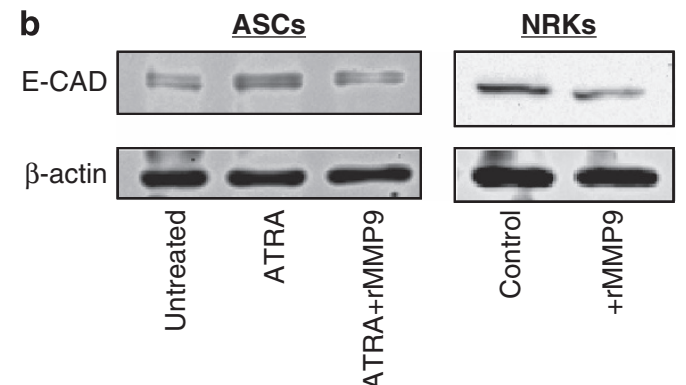

Figure 7 MMP9 is involved in ASC differentiation with ATRA. (a) In situ zymography for MMP9. The left column panel shows the increased gelatinolytic activity in ASCs cultured with ATRA and silenced at day 4 with $100 \mathrm{nM}$ antiLet-7e (ATRA + antiLet-7e) with respect to ASCs transfected with a control oligonucleotide (ATRA + CantimiR). The middle column panel indicates the results obtained when ASCs were incubated for $1 \mathrm{~h}$ with FITC-labeled DQ-gelatin. Green dots represent gelatinolytic activity of cultured cells. Results show an increase in gelatinolytic activity in silenced cells (ATRA + antiLet-7e). Magnification $\times 20$ (middle panel). Magnification $\times 40$ (right panel). A negative control was included by incubating the cells with $20 \mathrm{mM}$ EDTA. (b) In two different kinds of epithelial cells, epithelial differentiated ASCs (left panel) and in renal epithelial cell line NRK-52e after addition of recombinant MMPg $(2 \mu \mathrm{g} / \mathrm{ml}$ and incubated for $72 \mathrm{~h})$, western blot analysis of E-cadherin indicates a reduction in E-cadherin protein expression after MMP9 addition in the two cell lines. Equal loading was controlled by $\beta$-actin expression. A representative picture from four experiments is shown. ASCs treated with ATRA showed a significant increase in E-cadherin expression compared with untreated cells. This increase could be blocked by the addition of recombinant MMP9 (ATRA + MMP9). Addition of recombinant MMP9 to ASCs (ATRA + MMP9) or NRK cells ( + MMP9) decreased E-cadherin expression

Epithelial differentiation. For epithelial differentiation, ASCs (passage 3) were cultured with ATRA (Sigma-Aldrich) at a final concentration of $5 \mu \mathrm{M}$ as previously described. ${ }^{26}$ ATRA was dissolved in low glucose $(100 \mathrm{mg} / \mathrm{dll})$ DMEM medium supplemented with $10 \%$ FBS and antibiotics. Medium was replaced every 3 days during a total period of 11 days, followed by analysis of the resulting cell population and evaluation of the morphology.

miRNA transfection. Transfections of ASCs with $100 \mathrm{nM}$ let-7e (5'-UGAGG UAGGAGGUUGUAUAGUU-3'; Dharmacon, Madrid, Spain), 100 nM LNA-anti-let7e (5'-ACTATACAACCTCCTACCTC-3'; Exiqon, Vedbaek, Denmark) or the respective negative controls were carried out using Lipofectamine 2000 (Invitrogen, Madrid, Spain) according to the manufacturer's instructions. Medium was replaced after $6 \mathrm{~h}$ of transfection. Transfection times are described in Figure 4.

RNA extraction, reverse transcription mRNA and RT-PCR. Total RNA was extracted using TRIZOL reagent (Invitrogen). Then, $1 \mu \mathrm{g}$ of total RNA was reverse transcribed using an iScript CDNA synthesis kit (Bio-Rad, Hercules, CA, USA). The qRT-PCRs were performed using either TaqMan Universal PCR Master Mix (Applied Biosystems, Madrid, Spain) or iQ SYBR Green Supermix (Bio-Rad) depending on the primer used. Mouse primers provided by Applied Biosystems and Qiagen (Madrid, Spain) were previously validated (GAPDH, SIX2, PAX2, MMP9, WT1, WNT4, vimentin and megalin). CK18 (forward: $5^{\prime}$-CAGT ATGAAGCGCTGGCTCAGA-3', reverse: $5^{\prime}$-CGGCAGACTTGGTGGTGA-3') and E-CAD (forward: 5'-TGAAGAAGGAGGTGGAGAAGAAGA-3', reverse: 5'-TGGGA
GCCACATCATTTCGA-3') were bought from Invitrogen. Results were normalized to glyceraldehyde 3-phosphate dehydrogenase (GAPDH).

miRNA target site prediction. miRNA sequences were retrieved from the miRBase database and prediction of putative target mRNAs were analyzed using the miRWalk database. ${ }^{22}$

Reverse transcription and RT-PCR of microRNA. Total RNA was extracted as described before, and specific reverse transcription for miRNA mmu-let-7e and U6 small nuclear RNA (U6 snRNA) was performed using TaqMan microRNA Assay (Applied Biosystems). qRT-PCR was performed using the TaqMan Universal PCR Master Mix (Applied Biosystems) and primers were provided by Applied Biosystems. Results were normalized to U6 snRNA.

Immunocytochemistry. Immunocytochemistry was performed at the time when the differentiation markers reached their maximum expression (day 11).

Cells were fixed in $4 \%$ paraformaldehyde for $15 \mathrm{~min}$ at RT, washed twice with PBS and fixed in ice cold acetone for 5 min. ASCs were then blocked in $1 \%$ BSA in PBS-T for 30 min at RT, followed by overnight incubation at $4{ }^{\circ} \mathrm{C}$ with rabbit anticytokeratin 18 (Millipore, Darmstadt, Germany ). A non-immune immunoglobulin of the same isotype as the primary antibody was used as a control for immunocytochemistry (Millipore). The secondary antibody (goat biotinylated antirabbit IgG; Vector Laboratories) was incubated for $1 \mathrm{~h}$ at RT. Samples were stained using the avidin-peroxidase conjugate method for $1 \mathrm{~h}$. Cellular nuclei were counterstained with hematoxylin (Sigma-Aldrich). 
DAPI and phalloidin staining. DAPI and phalloidin staining was performed on day 11 , when the differentiation markers reached their maximum expression. Cells were washed with PBS, fixed in $4 \%$ paraformaldehyde for 10 min at RT and permeabilized with ice cold acetone for $5 \mathrm{~min}$. Cells were blocked in goat serum for $1 \mathrm{~h}$ at RT and then stained with Alexa Fluor 568 phalloidin (Invitrogen) in $1 \%$ PBS/BSA for $30 \mathrm{~min}$. Nuclei were visualized by the addition of DAPI solution $(0.2 \mu \mathrm{g} / \mathrm{ml})$ for $5 \mathrm{~min}$ at RT.

Dual luciferase reporter gene constructs. The vector constructs were commercially available and obtained from GeneCopoeia (Rockville, MD, USA): control vector (pEzX-MT01) and reporter construct containing the $3^{\prime} U T R$ of mouse MMP9 (pEzX-MT01-MMP9 $3^{\prime} U T R$ ). A deletion for the let-7e predicted binding site was obtained using the QuickChange II XL site-directed mutagenesis kit (Stratagene, Santa Clara, CA, USA). The correct sequences were confirmed by sequencing. PEzX-MT01 vectors use firefly luciferase as reporter gene under the control of a SV40 promoter and Renilla luciferase as tracking gene under the control of a CMV promoter.

Luciferase assay. A total of 90000 HEK293 cells (very easy to transfect) were used and transiently transfected with $0.1 \mu \mathrm{g}$ of vector constructs using Lipofectamine 2000 (Invitrogen) for 6 according to the manufacturer's instructions. Cells were co-transfected with $100 \mathrm{nM}$ miRNA let-7e or the respective negative control.

A total of 22000 ASCs were cultured in 12-well plates and differentiated for 1-11 days. Cells were transfected at different days of the culture with $1 \mu \mathrm{g}$ of vector constructs using Nanojuice transfection reagent (Novagen, Darmstadt, Germany) according to the manufacturer's instructions. Cells were co-transfected as indicated with $100 \mathrm{nM}$ miRNA let-7e, LNA-antiLet-7e or the respective negative controls. After transfection, cells were lysed with $1 \times$ passive lysis buffer (Promega, Madison, WI, USA) and luciferase activities were measured as light emission after addition of Renilla and Firefly luciferase substracts using a luminometer (Mithras, Berthold Technologies, Bad Wildbad, Germany). Firefly luciferase activity was normalized to Renilla luciferase activity in the cell lysate. The background obtained from cells treated with Lipofectamine but without the vector construct (mock-transfected cells) was subtracted from each experimental value.

Gelatin zymography. Supernatants of ASCs were collected $24 \mathrm{~h}$ after transfection with let-7e, and $1 \mathrm{ml}$ of each sample was used for gelatin zymography experiments to evaluate MMP9 activity. Supernatants were incubated with $50 \mu \mathrm{l}$ of gelatin-sepharose 4B (GE Healthcare, Barcelona, Spain) under constant shaking at $4^{\circ} \mathrm{C}$ for $1 \mathrm{~h}$ and centrifuged at $500 \times g$ for $2 \mathrm{~min}$ at $4{ }^{\circ} \mathrm{C}$. The resulting pellet containing gelatinases was washed by centrifugation at $500 \times g$ for $2 \mathrm{~min}$ at $4^{\circ} \mathrm{C}$ before separation of gelatinases with elution buffer by incubation at $4{ }^{\circ} \mathrm{C}$ under constant shaking for $30 \mathrm{~min}$. Gelatinase fraction (1:1) was dissolved in Laemmli sample buffer (Bio-Rad) and loaded on gels containing $8 \%$ acrylamide and porcine gelatin $(1 \mathrm{mg} / \mathrm{ml})$. After electrophoresis, gels were incubated with $250 \mathrm{ml}$ of buffer containing $50 \mathrm{mM}$ Tris- $\mathrm{HCl}, \mathrm{pH}, 7.5,10 \mathrm{mM} \mathrm{CaCl}_{2}$ and $0.02 \% \mathrm{NaN}_{3}$, for $24 \mathrm{~h}$ at $37^{\circ} \mathrm{C}$ in order to activate gelatinases. After incubation, gels were stained with $0.1 \%$ amido black (naphthol blue black, Sigma-Aldrich) in acetic acid/methanol/water $(1: 3: 6)$ for $30 \mathrm{~min}$. Then, gels were unstained by immersing in acetic acid/ methanol/water $(1: 3: 6)$ for $30 \mathrm{~min}$. To determine gelatinase activity, gels were scanned in a densitometer and relative activity levels were analyzed with digital image analysis using Quantity one 4.5.1 Software (Bio-Rad).

In situ zymography. In situ zymography was performed as previously described. ${ }^{46}$ ASCs were cultured with ATRA and incubated with $10 \mu \mathrm{g} / \mathrm{ml}$ of FITClabeled DQ-gelatin (Molecular Probes, Eugene, OR, USA) for $1 \mathrm{~h}$ at RT. Then, cells were washed with PBS and counterstained with Hoechst 33258 dye. Green FITC fluorescence indicative of gelatinase activity was observed using fluorescence microscopy (Leica, Solms, Germany). Negative control was performed using $20 \mathrm{mM}$ EDTA.

Recombinant MMP9 treatment. The role of MMP9 in E-CAD degradation was studied in mature epithelial cells such as NRK52E kidney tubular epithelia cells or in ASCs differentiated to epithelial cells.

NRK52E kidney tubular epithelial cells and ASCs after 8 days of differentiation were treated for 3 days with mouse recombinant MMP9 protein (Abcam, Cambridge, UK) as previously reported $(2 \mu \mathrm{g} / \mathrm{ml}),{ }^{36}$ in order to obtain samples at day 11 .
Western blot. ASCs were lysed in RIPA buffer $(150 \mathrm{mM} \mathrm{NaCl}, 1 \% \mathrm{NP}-40$, $0.1 \%$ SDS, $50 \mathrm{mM}$ Tris-base, $0.5 \%$ sodium deoxycholate containing $1 \mathrm{mM}$ PMSF and $5 \mathrm{mM}$ EDTA) at $4^{\circ} \mathrm{C}$. Protein concentration was measured using Bradford (Bio-Rad). Then, $20 \mu \mathrm{g}$ of total protein was electrophoresed on $10 \%$ SDSpolyacrylamide gels and transferred on nitrocellulose membranes. Membranes were incubated overnight at $4{ }^{\circ} \mathrm{C}$ with the following primary antibodies: anti-Ecadherin (Santa Cruz, Dallas, TX, USA) and anti- $\beta$-Actin (Sigma-Aldrich). Membranes were then incubated for $1 \mathrm{~h}$ at RT with horseradish peroxidaseconjugated anti-rabbit IgG (Sigma-Aldrich), followed by ECL detection. Equal protein loading was verified by immunoblotting for $\beta$-actin.

Statistical analysis. Data are expressed as means \pm S.D. Means of different groups were compared using one-way ANOVA. The Student-Newman-Keuls test was used for the evaluation of significant differences between groups. The existence of significant differences was assumed when $P<0.05$.

\section{Conflict of Interest}

The authors declare no conflict of interest.

Acknowledgements. We thank M Ángeles Muñoz for excellent technical support. This work was supported by Plan Nacional Project (SAF 2012-39947-CO2 to $\mathrm{GH}$ ) and Fondo de Investigación Sanitaria projects (FIS PS09/01288 and PI $12 / 00720$ to AS). AS is supported by the Miguel Servet contracting system (CP08/00138). MV is supported by a grant from IDIBAPS (beques de formació de personal investigador Institut d'Investigacions Biomèdiques August Pi i Sunyer).

1. Zuk PA, Zhu M, Ashjian P, De Ugarte DA, Huang JI, Mizuno H et al. Human adipose tissue is a source of multipotent stem cells. Mol Bio Cell 2002; 13: 4279-4295.

2. Gimble JM, Guilak F. Differentiation potential of adipose derived adult stem (ADAS) cells. Curr Top Dev Biol 2003; 58: 137-160.

3. Banas A, Teratani T, Yamamoto Y, Takeshita F, Quinn G, Okochi H et al. Adipose tissuederived mesenchymal stem cells as a source of human hepatocytes. Hepatology 2007; 46: 219-228

4. Ashjian PH, Elbarbary AS, Edmonds B, DeUgarte D, Zhu M, Zuk PA et al. In vitro differentiation of human processed lipoaspirate cells into early neural progenitors. Plast Reconstr Surg 2003; 111: 1922-1931.

5. Cao Y, Sun Z, Liao L, Han Q, Zhao RC. Human adipose tissue-derived stem cells differentiate into endothelial cells in vitro and improve postnatal neovascularization in vivo. Biochem Biophys Res Commun 2005; 332: 370-379.

6. Li K, Han Q, Yan X, Liao L, Zhao RC. Not a process of simple vicariousness, the differentiation of human adipose-derived mesenchymal stem cells to renal tubular epithelial cells plays an important role in acute kidney injury repairing. Stem Cells Dev 2010; 19: 1267-1275.

7. Baer PC. Adipose-derived stem cells and their potential to differentiate into the epithelial lineage. Stem Cells Dev 2011; 20: 1805-1816.

8. Horster MF, Braun GS, Huber SM. Embryonic renal epithelia: induction, nephrogenesis, and cell differentiation. Physiol Rev. 1999; 79: 1157-1191.

9. Lelongt B, Trugnan G, Murphy G, Ronco PM. Matrix metalloproteinases MMP2 and MMPg are produced in early stages of kidney morphogenesis but only MMP9 is required for renal organogenesis in vitro. J Cell Biol 1997; 136: 1363-1373.

10. Arnould C, Lelievre-Pegorier M, Ronco $P$, Lelongt $B$. MMP9 limits apoptosis and stimulates branching morphogenesis during kidney development. J Am Soc Nephrol 2009; 20: 2171-2180.

11. Chen JF, Mandel EM, Thomson JM, Wu Q, Callis TE, Hammond SM et al. The role of microRNA-1 and microRNA-133 in skeletal muscle proliferation and differentiation. Nat Genet 2006; 38: 228-233.

12. Zhao Y, Ransom JF, Li A, Vedantham V, von Drehle M, Muth AN et al. Dysregulation of cardiogenesis, cardiac conduction, and cell cycle in mice lacking miRNA-1-2. Cell 2007; 129: 303-317.

13. Krichevsky AM, Sonntag KC, Isacson O, Kosik KS. Specific microRNAs modulate embryonic stem cell-derived neurogenesis. Stem Cells 2006; 24: 857-864.

14. Liu Y, Huang T, Zhao X, Cheng L. MicroRNAs modulate the Wnt signaling pathway through targeting its inhibitors. Biochem Biophys Res Commun 2011; 408: 259-264.

15. Peter ME. Let-7 and miR-200 microRNAs: guardians against pluripotency and cancer progression. Cell Cycle 2009; 8: 843-852.

16. Tian Z, Greene AS, Pietrusz JL, Matus IR, Liang M. MicroRNA-target pairs in the rat kidney identified by microRNA microarray, proteomic, and bioinformatic analysis. Genome Res 2008; 18: 404-411.

17. Goff LA, Boucher S, Ricupero CL, Fenstermacher S, Swerdel M, Chase LG et al. Differentiating human multipotent mesenchymal stromal cells regulate microRNAs: prediction of microRNA regulation by PDGF during osteogenesis. Exp. Hematol 2008; 36: 1354-1369. 
18. Zhao C, Sun G, Li S, Lang MF, Yang S, Li W et al. MicroRNA let-7b regulates neural stem cell proliferation and differentiation by targeting nuclear receptor $T L X$ signaling. Proc Natl Acad Sci USA 2010; 107: 1876-1881.

19. Heinzelmann J, Henning B, Sanjmyatav J, Posorski N, Steiner T, Wunderlich $\mathrm{H}$ et al. Specific miRNA signatures are associated with metastasis and poor prognosis in clear cell renal cell carcinoma. World J Urol 2011; 29: 367-373.

20. Fu TY, Chang CC, Lin CT, Lai CH, Peng SY, Ko YJ et al. Let-7b-mediated suppression of basigin expression and metastasis in mouse melanoma cells. Exp Cell Res 2011; 317: 45-51.

21. Fu TY, Lin CT, Tang PC. Steroid hormone-regulated let-7b mediates cell proliferation and basigin expression in the mouse endometrium. J Reprod Dev 2011; 57: 627-635.

22. Dweep H, Sticht C, Pandey N, Gretz P. miRWalk-database: prediction of possible miRNA binding sites by "walking" the genes of three genomes. J Biomed Inform 2011; 44: 839-847.

23. Peister A, Mellad JA, Larson BL, Hall BM, Gibson LF, Prockop DJ. Adult stem cells from bone marrow (MSCs) isolated from different strains of inbred mice vary in surface epitopes, rates of proliferation, and differentiation potential. Blood 2004; 103: 1662-1668.

24. Baer PC, Bereiter-Hahn J, Missler C, Brzoska M, Schubert R, Gauer S et al. Conditioned medium from renal tubular epithelial cells initiates differentiation of human mesenchymal stem cells. Cell Prolif 2009; 42: 29-37.

25. Baer PC, Doring C, Hansmann ML, Schubert R, Geiger H. New insights into epithelial differentiation of human adipose-derived stem cells. J Tissue Eng Regen Med 2013; 7: 271-278.

26. Brzoska M, Geiger H, Gauer S, Baer P. Epithelial differentiation of human adipose tissue-derived adult stem cells. Biochem Biophys Res Commun 2005; 330: 142-150.

27. Bouchard M, Souabni A, Mandler M, Neubuser A, Busslinger M. Nephric lineage specification by Pax2 and Pax8. Genes Dev 2002; 16: 2958-2970.

28. Kobayashi A, Valerius MT, Mugford JW, Carroll TJ, Self M, Oliver G et al. Six2 defines and regulates a multipotent self-renewing nephron progenitor population throughout mammalian kidney development. Cell Stem Cell 2008; 3: 169-181.

29. Ryan G, Steele-Perkins V, Morris JF, Rauscher FJ 3rd, Dressler GR. Repression of Pax-2 by WT1 during normal kidney development. Development 1995; 12: 867-875.

30. Stark K, Vainio S, Vassileva G, McMahon AP. Epithelial transformation of metanephric mesenchyme in the developing kidney regulated by Wnt-4. Nature 1994; 372: 679-683.

31. Griesche N, Bereiter-Hahn J, Geiger H, Schubert R, Baer PC. During epithelial differentiation of human adipose-derived stromal/stem cells, expression of zonula occludens protein- 1 is induced by a combination of retinoic acid, activin- $A$ and bone morphogenetic protein-7. Cytotherapy 2012; 14: 61-69.

32. Liu J, Huang J, Lin T, Zhang C, Yin X. Cell-to-cell contact induces human adipose tissue-derived stromal cells to differentiate into urothelium-like cells in vitro. Biochem Biophys Res Commun 2009; 390: 931-936.

33. Bacallao R. The role of the cytoskeleton in renal development. Semin Nephrol 1995; 15 : 285-290.
34. Chandrasekaran K, Karolina DS, Sepramaniam S, Armugam A, Wintour EM, Bertram JF et al. Role of microRNAs in kidney homeostasis and disease. Kidney Int 2012; 81: 617-627.

35. Legallicier B, Trugnan G, Murphy G, Lelongt B, Ronco P. Expression of the type IV collagenase system during mouse kidney development and tubule segmentation. J Am Soc Nephrol 2001; 12: 2358-2369.

36. Zheng G, Lyons JG, Tan TK, Wang Y, Hsu TT, Min D et al. Disruption of E-cadherin by matrix metalloproteinase directly mediates epithelial-mesenchymal transition downstream of transforming growth factor-beta1 in renal tubular epithelial cells. Am J Pathol 2009; 175: 580-591.

37. Sternlicht MD, Werb Z. How matrix metalloproteinases regulate cell behavior. Annu Rev Cell Dev Biol 2001; 17: 463-516.

38. Nagase H, Woessner JF Jr. Matrix metalloproteinases. J Biol Chem 1999; 274 21491-21494.

39. Nthnick WB. Regulation of uterine matrix metalloproteinase- 9 and the role of microRNAs Semin Reprod Med 2008; 26: 494-940.

40. Ramos TV, Wang T, Maki CB, Pascual M, Izadyar F. Adipose stem cell side population in the mouse. J Tissue Eng Regen Med 2009; 3: 430-441.

41. Park SJ, Lee KW, Lim DS, Lee S. The sulfated polysaccharide fucoidan stimulates osteogenic differentiation of human adipose-derived stem cells. Stem Cells Dev 2012; 21: 2204-2211.

42. Yamamoto N, Akamatsu H, Hasegawa S, Yamada T, Nakata S, Ohkuma M et al. Isolation of multipotent stem cells from mouse adipose tissue. J Dermatol Sci 2007; 48: 43-52.

43. Maddox JR, Liao X, Li F, Niyibizi C. Effects of culturing on the stability of the putative murine adipose derived stem cells markers. Open Stem Cell J 2009; 1: 54-61.

44. Chamberlain G, Wright K, Rot A, Ashton B, Middleton J. Murine mesenchymal stem cells exhibit a restricted repertoire of functional chemokine receptors: comparison with human. PLoS One 2008; 3: e2934.

45. Futami I, Ishijima M, Kaneko H, Tsuji K, Ichikawa-Tomikawa N, Sadatsuki R et al. Isolation and characterization of multipotential mesenchymal cells from the mouse synovium. PLOS One 2012; 7: e45517.

46. Sans-Fons MG, Sole S, Sanfeliu C, Planas AM. Matrix metalloproteinase-9 and cell division in neuroblastoma cells and bone marrow macrophages. Am J Pathol 2010; 177: 2870-2885.

cc) (1) $(\Theta)$ Cell Death and Disease is an open-access journal (c) licensed under a Creative Commons Attribution-NonCommercialNoDerivs 3.0 Unported License. To view a copy of this license, visit http://creativecommons.org/licenses/by-nc-nd/3.0/ 